\title{
Evolutionary techniques applied to the optimal short-term scheduling of the electrical energy production
}

\author{
Alicia Troncoso , José C. Riquelme , Jesús S. Aguilar-Ruiz , \\ Jesús M. Riquelme Santos
}

\begin{abstract}
This paper presents an evolutionary technique applied to the optimal short-term scheduling ( $24 \mathrm{~h})$ of the electric energy production. The equations that define the problem lead to a non-convex non-linear programming problem with a high number of continuous and discrete variables. Consequently, the resolution of the problem based on combinatorial methods is rather hard. The required heuristics, introduced to assure the feasibility of the constraints, are analyzed, along with a brief description of the proposed genetic algorithm (GA). The GA is used to compute the optimal on/off status of thermal units and the fitness function is obtained by solving a quadratic programming problem by means of a standard non-linear Interior Point (IP) method. The results from real-world cases based on the Spanish power system are reported, which show the good performance of the proposed algorithm, taking into account the complexity and dimensionality of the problem. Finally, an IP algorithm is adapted to deal with discrete variables that appear in this problem and the obtained results are compared with that of the proposed GA.
\end{abstract}

Keywords: Genetic algorithms; Scheduling; Optimization; Feasibility; Interior point algorithms

\section{Introduction}

The optimal short-term scheduling of the electrical energy production [1] aims at determining which generating units should be online and the corresponding optimal generation of thermal and hydro

\footnotetext{
* Corresponding author.

E-mail addresses: atrolor@upo.es (A. Troncoso), riquelme@ lsi.us.es (J.C. Riquelme), jsagurui@upo.es (J.S. Aguilar-Ruiz), jsantos@us.es (J.M. Riquelme Santos).
}

units along the scheduling period, usually $24 \mathrm{~h}$, in order to minimize the expected total cost satisfying the forecasted system load. The scheduling task leads to a non-linear mixed-integer programming problem. Moreover, this problem is coupled in time by the maximum speed that generating units, specially thermal units, are able to change the produced energy (known as up and down ramps), and also by the topology of the hydroelectric power plants, with a delay in hours between the water of a reservoir being used and the availability of that water in the 
reservoirs downstream. A really large number of variables, both continuous and discrete variables, is needed to properly model this problem. Many approaches have been proposed for the resolution of this optimization problem, ranging from Dynamic Programming to Linear Mixed-Integer Programming or Lagrangian Relaxation [2], the latter being the most widely used optimization method in commercial programs. Genetic Algorithms (GAs) [3,4], a general-purpose stochastic search method based on the mechanics of natural selection, have also been successfully applied to the electrical energy scheduling problem since the adaptation is quite straightforward due to the combinatorial nature of this problem. In the last few years, the Interior-Point (IP) or Logarithmic-Barrier class of methods has become the preferred numerical approach to solve non-linear optimization problems, as a consequence of its enhanced capability to deal with inequality constraints $[5,6]$. Since the early developments, intended for Linear Programming problems, many improvements have been proposed in order to extend the application of IP methods to convex and non-convex non-linear problems. These refinements have to do with stepsize control [7], line-search techniques to force convergence to a local minimum from arbitrary starting points [8,9], use of trust regions [10], among others. However, despite these improvements, its performance on mixed-integer problems is frequently disappointing because the solution is far from the global optimum [11]. This has raised the interest in computationally expensive algorithms taken from the artificial intelligence area such as evolutionary methods [12], tabu search [13], particle swarm optimization [14], simulated annealing [15] or ant colony optimization [16]. Although convergence to the global optimum cannot be theoretically guaranteed, the ability to escape from local minima makes them an attractive choice for many applications $[17,18]$.

In this paper, a GA applied to the optimal shortterm $(24 \mathrm{~h})$ electrical energy production scheduling is presented. Some heuristics are included in order to assure the feasibility of the constraints that appear in the problem. Results from real-world cases based on Spanish power system are reported. The electric energy production scheduling presents a large number of variables and constraints, a non-convex non-linear objective function and integer and continuous variables. Thus, the main difficulty is to find feasible solutions and the novelty of the paper is addressed to improve the feasibility. The main contributions of the encoded GA can be stated as: a procedure to generate the initial population taking into account the ramp constraints; dynamic constraints of minimum limits on the hourly energy production of the thermal units to consider the starting and stopping periods as an alternative to the inclusion of other binary variables to model these states; a crossover operator adapted to the features of this problem leading to an adequate percentage of feasible individuals; and sparsity techniques and optimal ordering used to reduce the computational overhead. In spite of the sparsity techniques, a potential limitation of the GA is rather related to the CPU time when a complex topology of hydroelectric power plants is analyzed.

The paper is organized as follows: Section 2 presents the equations used to model the scheduling problem, leading to a non-linear mixed-integer programming problem with a large number of both continuous and discrete variables. In Section 3 a briefly description of the primal-dual IP algorithm is made. Section 4 introduces the proposed GA, and several implementation issues that are crucial to obtain feasible solutions are discussed. Finally, Section 5 reports some results obtained from realistic cases based on the Spanish power system, and the main conclusions of the paper are outlined.

\section{Formulation of the problem}

The objective of the scheduling problem is to determine the on/off state and the energy production of thermal and hydro units at each hour of the scheduling period, in order to minimize the total cost of the system satisfying the forecasted hourly demand and the technical constraints of thermal and hydro power plants.

The standard notation used for the scheduling of the electrical energy production problem is summarized in Table 1. This notation describes fixed parameters of the thermal and hydro units, indexes, number of elements and variables.

\subsection{Objective function}

The total energy production cost of the scheduling period is defined by 
Table 1

Definition of the data and variables of the problem

\begin{tabular}{|c|c|}
\hline \multicolumn{2}{|c|}{ Data or fixed parameters } \\
\hline$S U_{i}$ & The start-up cost of the thermal unit $i(€)$ \\
\hline$S D_{i}$ & The shut-down cost of the thermal unit $i(€)$ \\
\hline$C_{i}(\cdot)$ & Quadratic cost function of the thermal unit $i(€)$ \\
\hline$P_{i}^{m}$ & Lower bound of the hourly energy production of the thermal unit $i(\mathrm{MWh})$ \\
\hline$P_{i}^{M}$ & Upper bound of the hourly energy production of the thermal unit $i(\mathrm{MWh})$ \\
\hline $\mathrm{PH}_{h}^{m}$ & Lower bound of the hourly energy production of the hydro plant $h(\mathrm{MWh})$ \\
\hline$P H_{h}^{M}$ & Upper bound of the hourly energy production of the hydro plant $h(\mathrm{MWh})$ \\
\hline$V H_{h}^{m}$ & Lower bound of the water level of the reservoir $h$ in terms of energy (MWh) \\
\hline$V H_{h}^{M}$ & Upper bound of the water level of the reservoir $h$ in terms of energy (MWh) \\
\hline$U R_{i}$ & Upper bound of the up rate of the thermal unit $i(\mathrm{MWh} / \mathrm{h})$ \\
\hline$D R_{i}$ & Lower bound of the down rate of the thermal unit $i(\mathrm{MWh} / \mathrm{h})$ \\
\hline$W_{h}$ & Inflow of the reservoir $h$ in terms of energy $(\mathrm{MWh})$ \\
\hline$D_{t}$ & Energy demand at hour $t(\mathrm{MWh})$ \\
\hline$R_{t}$ & Generating capacity in reserve at hour $t(\mathrm{MWh})$ \\
\hline$D T_{i}$ & Number of hours that the unit $i$ must be shut-down after stopping \\
\hline$U T_{i}$ & Number of hours that the unit $i$ must be functioning after starting \\
\hline$d(k)$ & Water delay time between reservoir $k$ and the next reservoir downstream (in h) \\
\hline$n(k)$ & Next reservoir downstream regarding the reservoir $k$ \\
\hline \multicolumn{2}{|c|}{ Indexes and number of elements } \\
\hline$i$ & Thermal unit index \\
\hline$h$ & Hydro plant index \\
\hline$t$ & Hour index \\
\hline$n_{g}$ & Number of thermal units \\
\hline$n_{h}$ & Number of hydro plants \\
\hline$n_{t}$ & Number of hours of the scheduling period \\
\hline \multicolumn{2}{|c|}{ Variables } \\
\hline$P_{i, t}$ & Energy production of the thermal unit $i$ at hour $t(\mathrm{MWh})$ \\
\hline$U_{i, t}^{i, t}$ & On/off state of the thermal generator $i$ at hour $t$ \\
\hline$P H_{h, t}$ & Energy production of the hydro plant $h$ at hour $t(\mathrm{MWh})$ \\
\hline$V H_{h, t}$ & Stored energy of the reservoir $h$ at hour $t(\mathrm{MWh})$ \\
\hline
\end{tabular}

$$
\begin{aligned}
C_{T}= & \sum_{t=1}^{n_{t}} \sum_{i=1}^{n_{g}}\left[C_{i}\left(P_{i, t}\right)+S U_{i} \cdot U_{i, t} \cdot\left(1-U_{i, t-1}\right)\right. \\
& \left.+S D_{i} \cdot\left(1-U_{i, t}\right) \cdot U_{i, t-1}\right],
\end{aligned}
$$

where $n_{t}$ is the number of hours of the scheduling period, $n_{g}$ the number of thermal units, each having a quadratic cost function, $C_{i}\left(P_{i, t}\right)$, of the energy production, $P_{i, t} ; S U_{i}$ and $S D_{i}$ are, respectively, the startup and shut-down cost of the thermal generator $i$, and $U_{i, t}$ is a binary variable representing the on/off state of the thermal generator $i$ at hour $t$.

It can be observed that the total production cost is a sum of quadratic functions of the energy of each thermal generator if the state of each generator was previously stated by the GA. This is the case of the proposed technique because the on/off states are managed by the GA. Notice that the production cost is only due to the production of thermal generators $P_{i, t}$, i.e., generators that produce energy by burning a fuel or by atomic means. Hydro units provide free-of-charge energy $P H_{h, t}$ that is only subject to the availability of water in the corresponding reservoirs.

\subsection{Constraints}

The minimization of the objective function is subject to technical constraints, water balance in hydroelectric power plants and the associated reservoirs, and to the system energy demand and reserve balances:

- Maximum and minimum limits on the hourly energy production of the thermal and hydro generators,

$$
\begin{aligned}
& P_{i}^{m} \leqslant P_{i, t} \leqslant P_{i}^{M}, \quad i=1, \ldots, n_{g}, t=1, \ldots, n_{t}, \quad(2) \\
& P H_{h}^{m} \leqslant P H_{h, t} \leqslant P H_{h}^{M}, \quad h=1, \ldots, n_{h}, t=1, \ldots, n_{t},
\end{aligned}
$$


where $n_{h}$ is the number of hydro plants, $P H_{h, t}$ the energy production of hydro plant $h$ at hour $t$, and $P_{i}^{m}, P_{i}^{M}, P H_{h}^{m}$ and $P H_{h}^{M}$ are the limits on the hourly energy production of the thermal unit $i$ and hydro plant $h$, respectively.

Eq. (2) cannot be fulfilled when thermal generators are either starting or stopping, as starting and stopping periods begin, respectively, when the corresponding state changes to $\mathrm{ON}$ or $\mathrm{OFF}$. In order to avoid this problem, this equation is modified for thermal units that are either being started-up or shut-down,

$0 \leqslant P_{i, t} \leqslant P_{i}^{M}, \quad i=1, \ldots, n_{g}, t=1, \ldots, n_{t}$.

Moreover, the energy produced by thermal units during periods of shutting-down $\left(U_{i, t}=0\right)$ is out of the optimal scheduling. Consequently, penalty terms proportional to this energy are added to the objective function as follows:

$C_{T}^{\prime}=C_{T}+\sum_{t=1}^{n_{t}} \sum_{i=1}^{n_{g}} C_{p} \cdot P_{i, t} \cdot\left(1-U_{i, t}\right)$.

- Maximum up and down ramps of thermal units. The thermal units can not increase or decrease the production of energy at consecutive hours by more than a given maximum rate,

$$
\begin{aligned}
& -D R_{i} \leqslant P_{i, t}-P_{i, t-1} \leqslant U R_{i}, \quad i=1, \ldots, n_{g}, \\
& \quad t=1, \ldots, n_{t},
\end{aligned}
$$

where $U R_{i}$ y $D R_{i}$ are, respectively, the maximum up and down rates of the thermal generator $i$, usually known as ramp limits.

- Limits on the available water. The hydro units use water to generate electrical energy and water is a limited resource. Thus, the energy produced by a hydro unit is limited by the volume of available water in the associated reservoir. In consequence, reservoir levels are subject to capacity limits,

$$
\begin{aligned}
& V H_{h}^{m} \leqslant V H_{h, t} \leqslant V H_{h}^{M}, \quad h=1, \ldots, n_{h}, \\
& \quad t=1, \ldots, n_{t},
\end{aligned}
$$

where $V H_{h, t}$ is the stored energy of reservoir $h$ at hour $t$, corresponding to the hydro unit $h ; V H_{h}^{m}$ and $V H_{h}^{M}$ are respectively the minimum and maximum limits on the stored energy imposed by the maximum and minimum possible water level of reservoir $h$.
- Hydraulic coupling between reservoirs. Time coupling exits due to cascaded reservoirs, since the water used to produce energy in a hydro unit will be available later to the next hydraulic unit downstream with a certain delay, obviously when the water has arrived to the corresponding reservoir.

$$
V H_{h, t}=V H_{h, t-1}-P H_{h, t}+\sum_{n(k)=h} P H_{k, t-d(k)}+W_{h},
$$

where $d(k)$ is the water delay time in hours between reservoir $k$ and the next reservoir downstream, $n(k)$, that is supposed to be reservoir $h$, and $W_{h}$ is the natural inflow of reservoir $h$.

- The total hourly energy production must be equal the total energy demand at that hour, $D_{t}$, which has been previously forecasted.

$$
\sum_{i=1}^{n_{g}} P_{i, t} \cdot U_{i, t}+\sum_{h=1}^{n_{h}} P H_{h, t}=D_{t}, \quad t=1, \ldots, n_{t} .
$$

- The total energy that can be produced at each hour must exceed the forecasted demand by a specified amount, $R_{t}$, i.e., the generating capacity in reserve to be used if an unexpected event such as the failure of a plant or a large error on the forecasted demand happens.

$$
\begin{gathered}
\sum_{i=1}^{n_{g}} P_{i}^{M} \cdot U_{i, t}+\sum_{h=1}^{n_{h}} P H_{h}^{M} \geqslant D_{t}+R_{t}, \\
t=1, \ldots, n_{t} .
\end{gathered}
$$

- Minimum up and down times of thermal units. The minimum up time, $U T_{i}$, is the minimum number of hours that the unit $i$ must be functioning after starting. Besides, the minimum down time, $D T_{i}$, is the minimum number of hours that the unit $i$ must be shut-down after stopping.

$$
\sum_{k=0}^{D T_{i}-1}\left(1-U_{i, t+k}\right) \geqslant D T_{i}
$$

if unit $i$ is shut-down at hour $t$

$$
\sum_{k=0}^{U T_{i}-1} U_{i, t+k} \geqslant U T_{i}
$$

if unit $i$ is started at hour $t$.

Start-up and shut-down costs of realistic cases tend to reduce the number of shut-downs and start-ups to a minimum, making the minimum-time constraints useless in most cases. Moreover, the inclusion of hydraulic generation facilitates the fulfillment of the thermal unit constraints because 
the hydro units are faster in response and produce energy at no cost, i.e., the hydraulic energy will be strategically distributed among the hours of the scheduling horizon in order to avoid the starting of more thermal units than the strictly required.

As an example, Table 2 shows the number of constraints, binary and continuous variables of the above problem for a test system comprising 49 thermal units, two hydro units and the scheduling horizon embracing $24 \mathrm{~h}$.

\section{Primal-dual IP algorithm}

Among the distinctive features of the above optimization problem, the most important are: large number of variables and constraints in practical cases; non-convexity of the objective function; and presence of integer and continuous variables. The IP methods only can be applied when all the variables of the problem are continuous. An alternative frequently used in practice consists in relaxing the discrete nature of $U_{i, t}$ and imposing instead the next constraint:

$0 \leqslant U_{i, t} \leqslant 1$

This leads to a simplified model without discrete variables which requires that a heuristic procedure be applied during or at the end of the iterative process in order to determine the best integer value for every $U_{i, t}$. Thus a mixed-integer programming problem is considered from a continuous perspective. In addition, every discrete variable can be handled as a continuous variable provided that the following quadratic constraint is added:

$U_{i, t} \cdot\left(1-U_{i, t}\right)=0$.

However, this procedure increases the non-linearity and non-convexity of the initial problem.

Based on the above comments and practical experience, the following procedure has been chosen to solve the optimal short-term scheduling of the electrical energy production.

Table 2

Dimension of the problem for a test system

\begin{tabular}{lll}
\hline Number of constraints & \multicolumn{2}{l}{ Number of variables } \\
\cline { 2 - 3 } & Binary & Continuous \\
\hline$\left(2 \cdot n_{g}+3 \cdot n_{h}+2\right) \cdot n_{t}+2 \cdot n_{g}$ & $n_{g} \cdot n_{t}$ & $\left(n_{g}+2 \cdot n_{h}\right) \cdot n_{t}$ \\
2642 & 1176 & 1272 \\
\hline
\end{tabular}

(1) The problem is solved adding the constraint (Eq. (13)).

(2) Using the obtained solution in the before step to initialize the IP algorithm, solve the problem including the constraint (Eq. (14)).

A brief description of a classical IP algorithm is provided next. This method introduces auxiliary positive slack variables in order to turn inequality restrictions into equality constraints:

$x_{j} \leqslant \bar{x}_{j} \leadsto x_{j}+s_{j}=\bar{x}_{j} \quad s_{j} \geqslant 0$,

where $x_{j}$ represents any variable subject to a limit, and $s_{j}$ is the corresponding positive slack variable.

In order to guarantee the positiveness of the slack variables, logarithmic penalty terms are included in the objective function by means of a penalty factor $\mu$ that is progressively reduced throughout the iterative process [5].

$f^{\prime}\left(x_{j}, s_{j}, \mu\right)=f\left(x_{j}\right)-\mu \sum_{j} \ln s_{j}$.

The main steps of the IP algorithm are the following:

(1) Initialize the variables so that the slack variables are positive.

(2) Initialize the penalty factor $\mu$ so as to make the logarithmic terms dominate over the original objective function.

(3) The minimization of the corresponding Lagrangian function is performed by solving the non-linear optimality equations using an one-step Newton's algorithm, and the optimal increment of primal and dual variables is computed.

(4) The step-length $\alpha$ is reduced, if necessary, so that the slack variables remain positive. Lagrange multipliers associated to equality constraints arising from the introduction of auxiliary slack variables must also remain positive because optimality conditions lead to equations of the form:

$s_{j} \cdot z_{j}=\mu$,

where $z_{j}$ is the Lagrange multiplier associated with the slack variable $s_{j}$.

(5) Update primal and dual variables taking into account the necessary step-length limitation.

(6) Reduce the penalty factor $\mu$. The proportional relationship between the penalty factor and the duality gap (dugap) defined by Eq. (17) 
provides the most common approach to reduce this penalty factor:

$$
\mu=\gamma \cdot \frac{\sum_{j=1}^{n_{l}} s_{j} \cdot z_{j}}{n_{l}},
$$

where $\gamma \leqslant 1$ and $n_{l}$ is the number of inequality constraints of the original problem. Steps 3-6 are iteratively repeated until optimality conditions are satisfied and the penalty coefficient $\mu$, and consequently the average duality gap, is small enough. More sophisticated versions of steps 3 and 4, including line searches, modified Hessians, etc. [8-10] could be needed in the non-convex case, in order to avoid divergence or convergence to unacceptable points. However, such refinements have not been actually implemented because the behaviour of the IP method has proven good enough for the application tested.

The application of Newton's method to solve the non-linear optimality equations yields a very large, sparse linear system, specially when ramp and hydraulic couplings are considered. Consequently, sparsity techniques and optimal ordering [19] must be used to reduce the computational overhead.

Fig. 1 shows the fill-ins generated when solving a small example (five hydro plants, five thermal plants and a 5-hour scheduling horizon), with and without optimal ordering. It can be noted that the fill-ins are reduced a $50 \%$ approximately when an optimal ordering is made. Table 3 shows relative execution times of the IP algorithm for two realistic problems (73 thermal plants, $24 \mathrm{~h}, 8$ and 30 reservoirs, respectively). As can be noticed, execution times grow considerably with the number of variables, specially when standard ordering is performed. Finally, Fig. 2 shows the relative overhead of the different processes comprising the IP algorithm.

\section{The proposed genetic algorithm}

As presented in the previous section, the optimal scheduling of the electric energy production is a non-linear, non-convex, combinatorial, mixed-integer and very large problem. Hence, there is no technique that would always lead to the optimal solution of the problem for realistic cases. In the last years, techniques based on heuristics, dynamic programming, linear mixed-integer programming and lagrangian relaxation have been applied to this particular problem. Techniques based on heuristics rely on simple rules that depends on the knowledge of power plant operators. Constraints of realistic problems are not properly modelled by dynamic programming approaches, and the number of required states increases exponentially, thus leading to excessive computation times. Linear programming approaches cannot properly model neither the non-linear objective function nor the non-linear constraints, and crude approximations are required. Finally, the use of heuristic techniques is required by lagrangian relaxation approaches to calculate feasible solutions, deteriorating the quality of the obtained solutions.

Consequently, new methods are still needed to obtain more optimal solutions to realistic problems. In this paper, a GA $[20,21]$ has been used to solve the scheduling problem due to its ability to deal with non-linear functions and integer variables.

The proposed GA algorithm is used to compute the optimal on/off states of thermal units, i.e., the binary variables, while the optimal continuous variables, i.e., the hourly energy production of hydro and committed thermal units, are calculated solving a typical quadratic programming problem by a classical IP optimization algorithm in which the on/off states of thermal units are known.

Convergence characteristics of GA depend on several key implementation issues that are discussed in the rest of this section.

\subsection{Codification of the individuals}

Each individual is represented by the on/off states of thermal generators during the scheduling period. Thus, individuals are represented by $0 / 1$ matrices, with columns corresponding to time scheduling intervals and rows associated with thermal units. If the element $(i, j)$ is equal to one, the state of thermal unit $i$ during time interval $j$ is on. Similarly, if the element $(i, j)$ is equal to zero, the state of thermal unit $i$ during time interval $j$ is off.

Fig. 3 shows the representation of a certain individual of the population. It can be observed that the thermal unit 1 is on from $1 \mathrm{am}$ to $4 \mathrm{am}$ and the rest of hours is off; the thermal unit 2 is off from 1 am to $6 \mathrm{am}$ and the rest of hours is on; the thermal unit 3 is on during all scheduling horizon; the thermal unit 4 is only on from $11 \mathrm{am}$ to $4 \mathrm{pm}$, etc.

\subsection{Initial population}

Up and down ramp constraints of thermal units (Eq. (6)) are a key factor in the convergence of the 

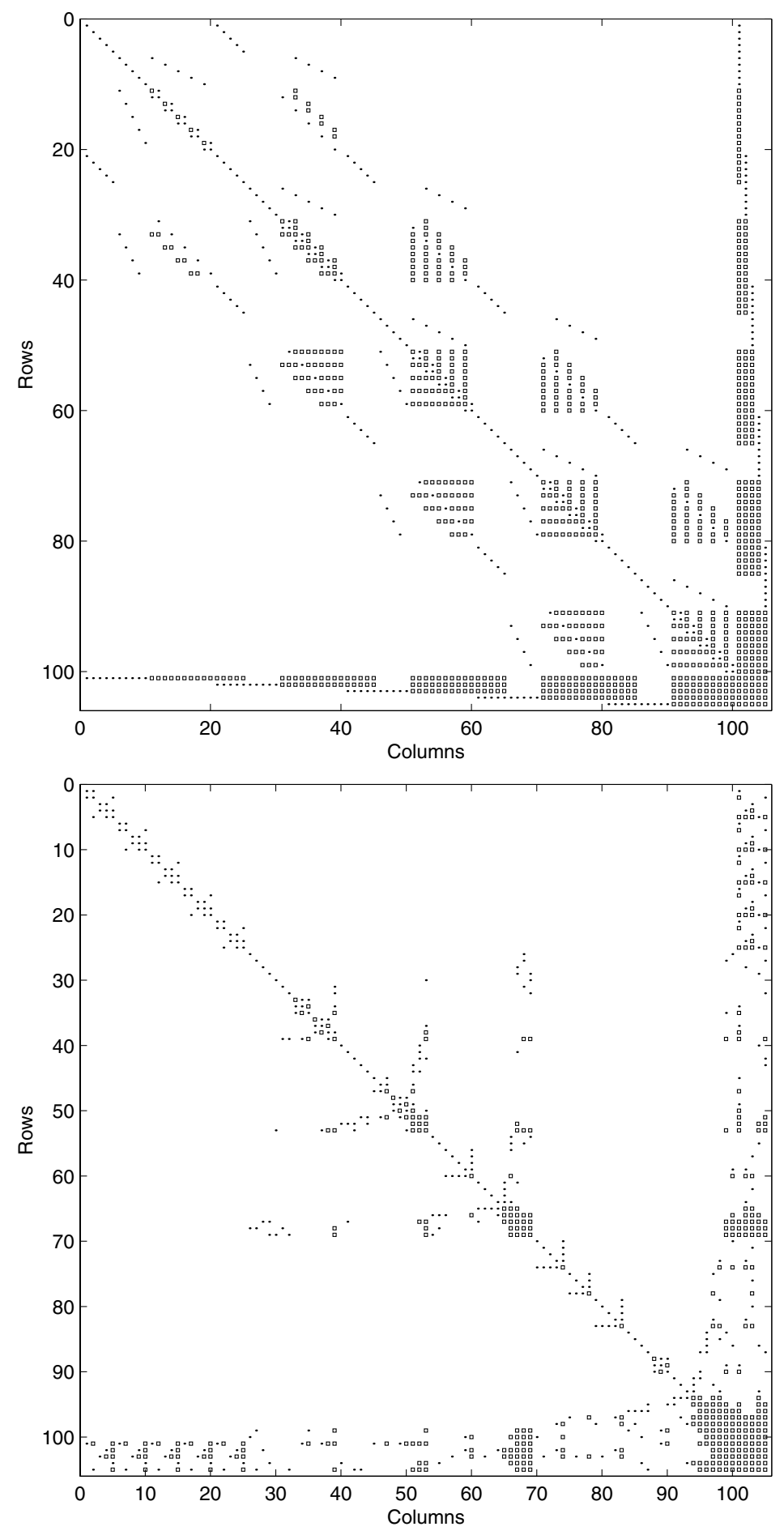

Fig. 1. Structure of the linear system including fill-ins.

GA: if the initial population is strictly randomly selected, ramp constraints lead to many infeasible individuals in the initial generation, which makes successive generations suffer from poor diversity, and the GA may converge prematurely. To assure that the initial population contains an adequate 
Table 3

Relative execution time and iteration number.

\begin{tabular}{llllll}
\hline \multirow{2}{*}{ Number of variables } & \multicolumn{2}{c}{ Natural ordering } & & \multicolumn{2}{c}{ Optimal ordering } \\
\cline { 2 - 3 } & Iterations & Time & & Iterations & Time \\
\hline 2376 & 33 & 4.00 & & 34 & 1.00 \\
3960 & 27 & 140.98 & & 34 & 2.45 \\
\hline
\end{tabular}

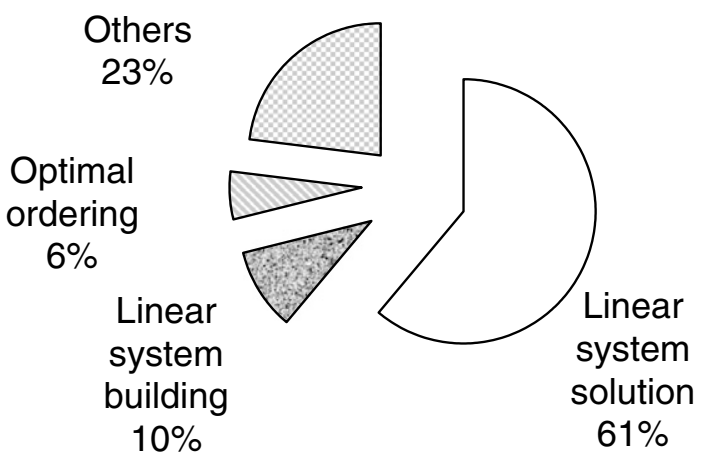

Fig. 2. Relative overhead of the different processes.

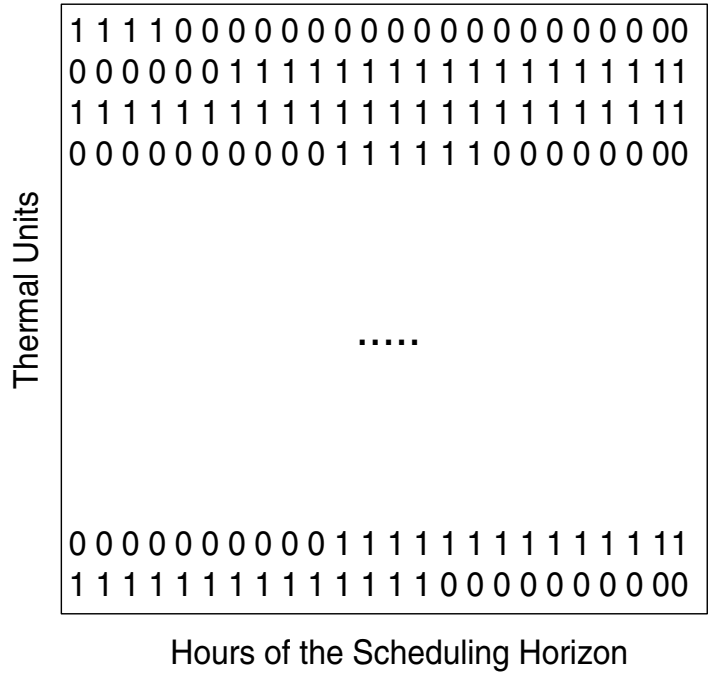

Fig. 3. Representation of an individual of the population.

percentage of feasible individuals, initial on/off schedulings are randomly selected but modified to account for the minimum start-up and shut-down times imposed by ramp constraints. For example, if generator $g$, with a maximum down ramp equal to $100 \mathrm{MWh}$, is on at hour 3 producing an energy of $400 \mathrm{MWh}$, this generator would require 4 hours to shut-down and, consequently, the generator at hours 4,5 and 6 should be on. The state $U_{g, 3}$ is strictly randomly generated but the states for the following hours, $U_{g, 4}, U_{g, 5}$ and $U_{g, 6}$, are given by

$U_{g, 3}=1 \Rightarrow U_{g, 4}=U_{g, 5}=U_{g, 6}=1$.

\subsection{Fitness function}

The fitness function evaluates the quality of an individual of the population. In this case, the function is the inverse of the total production cost of the individual. The total production cost is obtained solving a quadratic programming problem by using a non-linear Interior Point method [22,23]. An extra-high-cost fictitious generator is included to satisfy the system demand (Eq. (9)). This fictitious generator generates the necessary energy that the rest of generators cannot produce to satisfy the demand of the customers. A penalty term proportional to the deficit in reserve requirements is added in the cost function aiming at satisfying the reserve constraint. Penalty terms only apply to infeasible individuals, which are consequently eliminated throughout the evolutionary process.

\subsection{Selection operator}

To produce a new generation, parents are randomly selected using a roulette wheel selection technique that selects the best individuals for reproduction. The probability of a particular individual being selected is in proportion to its fitness function, taking into account that the total generation cost, including possible penalizations, is being minimized. The individuals chosen to be parents are included in the following generation.

\subsection{Crossover operator}

Offspring is obtained by adding the binary strings that results from random partitions of each row, as shown in Fig. 4a. A column-partitioning procedure may also be applied (Fig. 4b). This crossover operator is a particular case of the multi-point crossover operator where the number of points is equal to the number of rows or columns, respectively.

As rows are associated with the thermal units, the first approach yields mainly the infeasibility of new individuals in terms of minimum up and down times (Eqs. (11) and (12)), while the second approach has an effect bigger on the constraint of the demand (Eq. (9)) and the reserve (Eq. (10)). This above statement is shown in the next example. Let be a test 


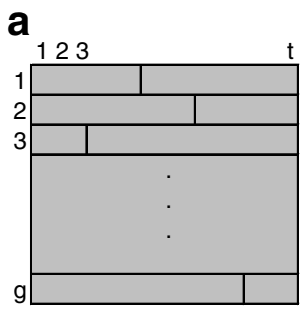

PARENTS

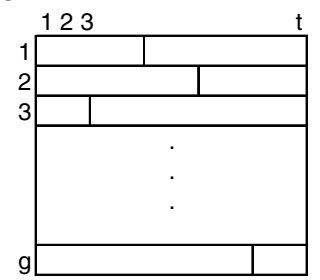

CHILDREN

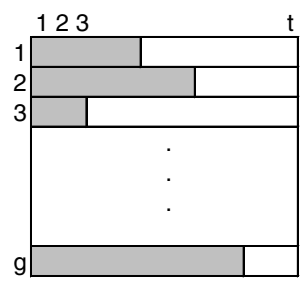

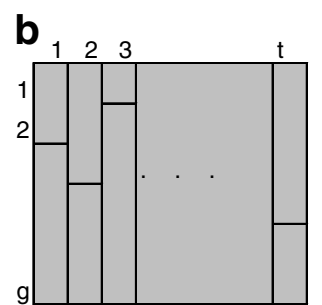

PARENTS

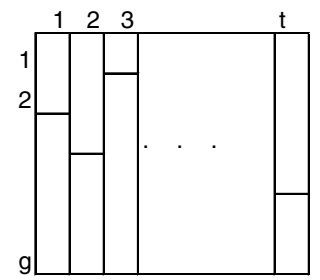

CHILDREN
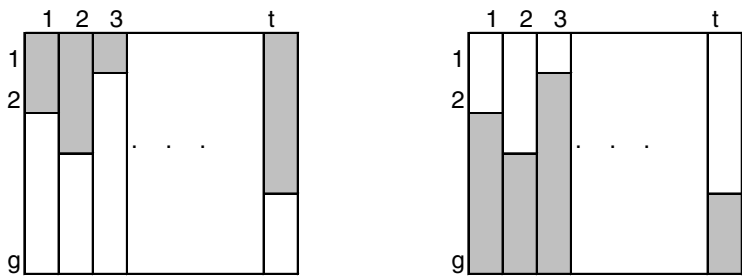

Fig. 4. Crossover Operator: (a) random partitions of rows and (b) random partitions of columns.

system comprising four thermal units and a 4-hour scheduling horizon. The minimum up and down time are 2 hours for all thermal units. Two individuals selected to be parents are represented in Fig. 5. Fig. 6 shows the children obtained using a crossover operator by rows from the random partition $(1,2,1,2)$. It can be observed that the children do not satisfy the Eqs. (11) and (12) (generator three) while the Eqs. (9) and (10) can be feasible. Fig. 7 shows the children obtained using a crossover operator by columns from the same partition. It can be observed that the individual on the right does not satisfy the Eqs. (9) and (10) since all generators are off at hours 3 and 4 . However, in this case the Eqs. (11) and (12) are fulfilled.

\begin{tabular}{l|llll|}
\multicolumn{1}{l}{} & $\mathrm{h} 1$ & $\mathrm{~h} 2$ & $\mathrm{~h} 3$ & $\mathrm{~h} 4$ \\
\cline { 2 - 4 } g1 & 1 & 1 & 1 & 1 \\
g2 & 1 & 1 & 0 & 0 \\
g3 & 0 & 0 & 0 & 0 \\
g4 & 1 & 1 & 0 & 0 \\
\cline { 2 - 4 } & &
\end{tabular}

\begin{tabular}{lrrrr|}
\multicolumn{1}{c}{} & \multicolumn{1}{c}{$\mathrm{h} 1$} & $\mathrm{~h} 2$ & $\mathrm{~h} 3$ & $\mathrm{~h} 4$ \\
\cline { 2 - 5 } g1 & 1 & 1 & 0 & 0 \\
g2 & 1 & 1 & 0 & 0 \\
g3 & 1 & 1 & 1 & 1 \\
g4 & 0 & 0 & 1 & 1 \\
\cline { 2 - 5 } & & &
\end{tabular}

Fig. 5. Parents.

\begin{tabular}{l|rrrr}
\multicolumn{1}{c}{} & \multicolumn{1}{c}{$\mathrm{h} 1$} & $\mathrm{~h} 2$ & $\mathrm{~h} 3$ & $\mathrm{~h} 4$ \\
\cline { 2 - 5 } g1 & 1 & 1 & 0 & 0 \\
g2 & 1 & 1 & 0 & 0 \\
g3 & 0 & 1 & 1 & 1 \\
g4 & 1 & 1 & 1 & 1 \\
\cline { 2 - 4 } & & &
\end{tabular}

\begin{tabular}{l|llll|}
\multicolumn{1}{c}{} & \multicolumn{1}{c}{ h1 } & h2 & h3 & h4 \\
\cline { 2 - 5 } g1 & 1 & 1 & 1 & 1 \\
g2 & 1 & 1 & 0 & 0 \\
g3 & 1 & 0 & 0 & 0 \\
g4 & 0 & 0 & 0 & 0 \\
\cline { 2 - 5 } & & &
\end{tabular}

Fig. 6. Children by partitions of rows.

\begin{tabular}{lrrrr|}
\multicolumn{1}{c}{} & $\mathrm{h} 1$ & $\mathrm{~h} 2$ & $\mathrm{~h} 3$ & $\mathrm{~h} 4$ \\
\cline { 2 - 5 } g1 & 1 & 1 & 1 & 1 \\
g2 & 1 & 1 & 0 & 0 \\
g3 & 1 & 1 & 1 & 1 \\
g4 & 0 & 0 & 1 & 1 \\
\cline { 2 - 4 } & & &
\end{tabular}

\begin{tabular}{l|rrrr|}
\multicolumn{1}{c}{} & \multicolumn{1}{c}{ h1 } & h2 & h3 & h4 \\
\cline { 2 - 5 } g1 & 1 & 1 & 0 & 0 \\
g2 & 1 & 1 & 0 & 0 \\
g3 & 0 & 0 & 0 & 0 \\
g4 & 1 & 1 & 0 & 0 \\
\cline { 2 - 5 } & & &
\end{tabular}

Fig. 7. Children by partitions of columns.

The crossover probability has been set to one, i.e., two individuals that have been selected to be parents are always combined to obtain a new individual.

In the final version of the GA, the crossover by rows has been chosen because start-up and shutdown costs of realistic cases, along with the inclusion of hydraulic generation, tend to reduce the number of shut-downs and start-ups to a minimum, making the minimum-time constraints useless in most cases. All the rows are always combined to obtain a new individual, though probabilities might have been used to determine which rows should be combined.

\subsection{Mutation operator}

After the crossover process, the individuals of the population are mutated to introduce some new genetic material according to a pre-defined mutation probability $p$. Consequently, the percentage of mutated individuals of a generation is equal to $100 p \%$. The mutation of an individual means the mutation of an only gene. The gene to be mutated 
is represented by a randomly selected generator and time interval, element $(i, j)$ of the matrix representing a particular individual. The mutation of a gene implies changing the state on/off of the generator.

\section{Test results}

The GA algorithm have been applied to several realistic cases based on the Spanish generation system, comprising 49 thermal units and one equivalent hydraulic generator, the scheduling horizon embracing 24 hours. Hourly system demand corresponds to a working day.

The main parameters of the implemented GA are as follows: 5000 for the maximum number of generations; 100 for the size of the population; 1 for the probability of crossover and 0.1 for the probability of mutation. To avoid any misinterpretation of the experimental results, the GA has been applied 10 times to the optimal scheduling electrical energy production.

Fig. 8 shows the evolution of the fittest individual cost and the average cost of the generation through-
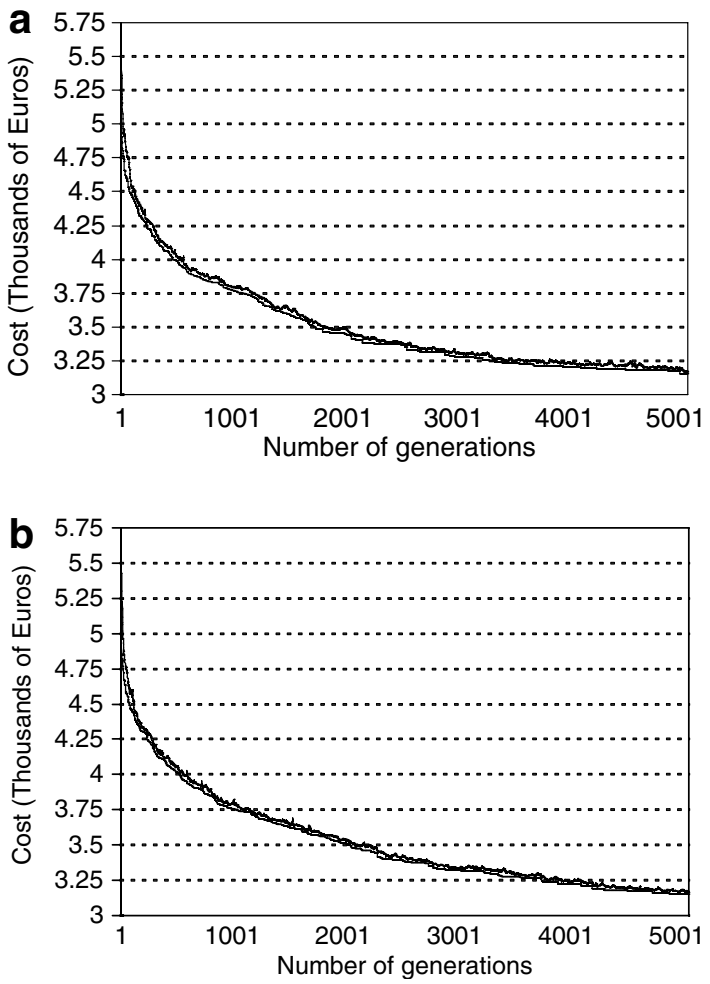

Fig. 8. Evolution of the best individual and average costs: (a) reserve requirements considered and (b) no reserve requirements considered. out the evolutionary process for 10 runs, with and without reserve requirements (Fig. 8a and b, respectively). It can be noticed that the initial population improves its quality throughout the generations. This fact is due to the adopted heuristics to generate the initial population taking into account the ramp constraints and the modified constraints (Eq. (4)) to assure the feasibility of the solutions at starting and stopping periods. Obviously, reserve requirements lead to higher operating costs, both in the best solution (3152.71 and 3109.51 thousands of Euros, respectively) and in the average (3170.90 and 3132.02 thousands of Euros, respectively). To make this fact more clear, the evolution of the last generations is depicted in Fig. 9.

Fig. 10 presents the optimal thermal and hydraulic generation, along with the evolution of the marginal cost during the scheduling period. The marginal cost represents the increment of cost when the system demand increases in one MWh, i.e., the hourly cost of the energy. Ramp constraints are only included in the second case (Fig. 10b). Note that, when ramps are considered, a higher cost,
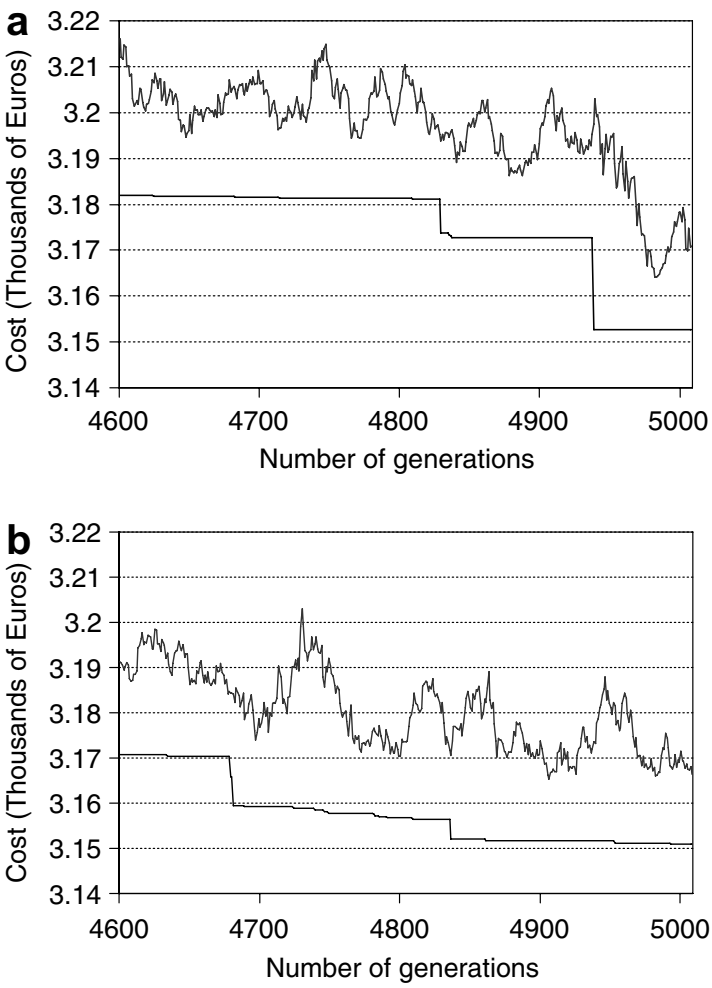

Fig. 9. Evolution of the best individual and average costs: (a) reserve requirements considered and (b) no reserve requirements considered. 

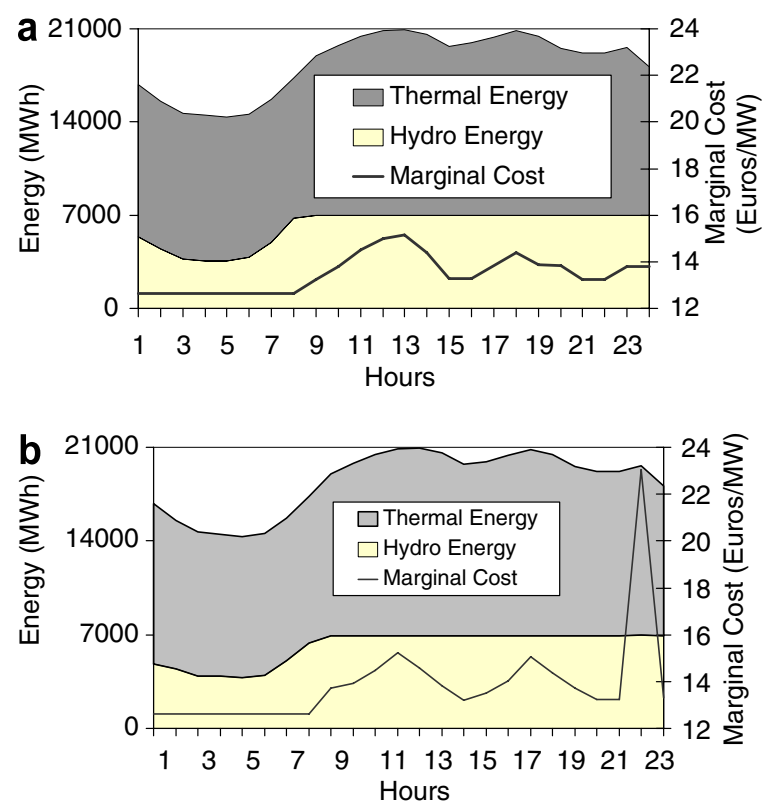

Fig. 10. Optimal thermal and hydraulic generation: (a) no ramp constraints considered and (b) ramp constraints considered.

fast-response generator is needed at hour 22 to satisfy a small peak of demand. As expected, the total operating cost is higher when ramps are included (3119.1 and 3109.5 thousands of Euros, respectively). It can be observed that the marginal cost has a similar shape to the electric energy demand. This means that the electric energy production is more expensive during the peak hours, i.e., hours with higher demand. The strategy followed by the GA is the optimal strategy. As the hydraulic generator does not have associated costs (hydraulic terms do not appear in the objective function (Eq. (1)), the hydraulic generator produces the maximum energy (7000 MW) in most of the hours of the scheduling period and the rest of the energy demand is fulfilled by the produced energy for the thermal generators.

Fig. 11 shows the optimal scheduling of a thermal generator, ignoring its ramp constraints (Fig. 11a) and considering them (Fig. 11b). Notice that ramps modify the optimal scheduling when the generator is starting and stopping. The penalty term imposed to the objective function when $U_{i, t}=0$ (Eq. (5)), forces the generator to adjust its output to the least possible value compatible with the ramp constraint (hours 15 and 16). Moreover, the minimum power of the thermal generators is equal to zero at starting and stopping periods.
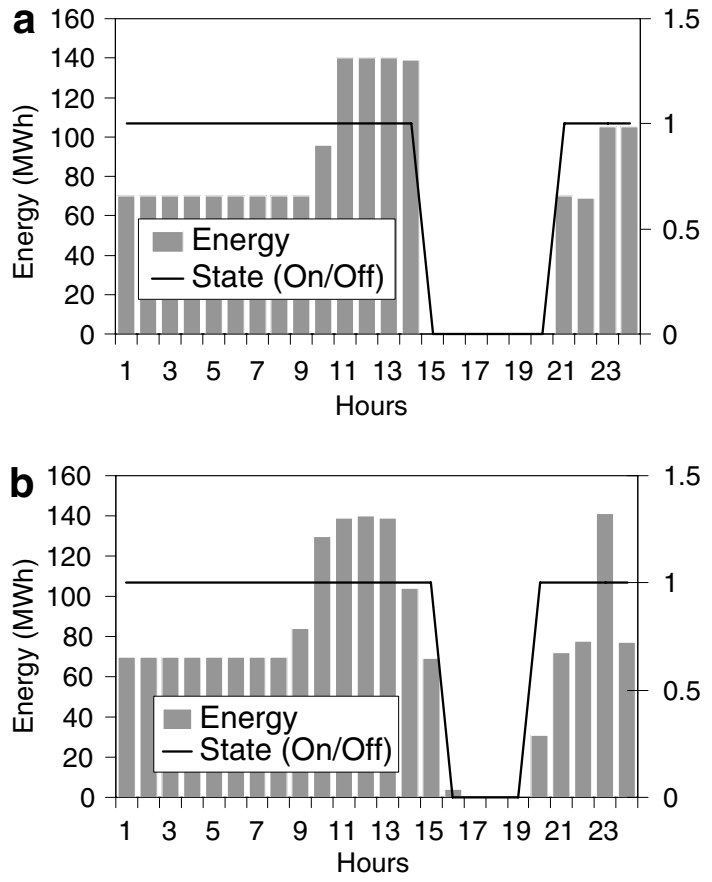

Fig. 11. Optimal scheduling of a thermal generator: (a) no ramp constraints considered and (b) ramp constraints considered.

Due to these considerations in these periods, a larger number of feasible individuals is obtained. Similar considerations apply when the generation is starting (hours 20 and 21).

Finally, Fig. 12 shows the solution provided by the proposed GA applied to the optimal scheduling of 49 thermal units and two cascaded reservoirs with a delay of $10 \mathrm{~h}$ and all the energy initially stored in the upstream reservoir. Note that the downstream reservoir 2 cannot start producing until water released by generator 1 arrives. As can be noticed, the total available hydraulic energy cannot be used. This fact is due to the hydraulic constraints and to the maximum power of generators. It is important to use the total available hydraulic energy since the hydro plants do not have associated costs as the fuel is water. Consequently, the optimal strategy is to consume all the hydraulic energy and the rest of the energy demand is completed by the produced energy for the thermal generators.

The performance of the GA is then compared to the IP algorithm described before for two cases: a small test system comprising five thermal units and the above system based on the Spanish generation system without hydro units. The ramps and the reserve requirements have been included in both 

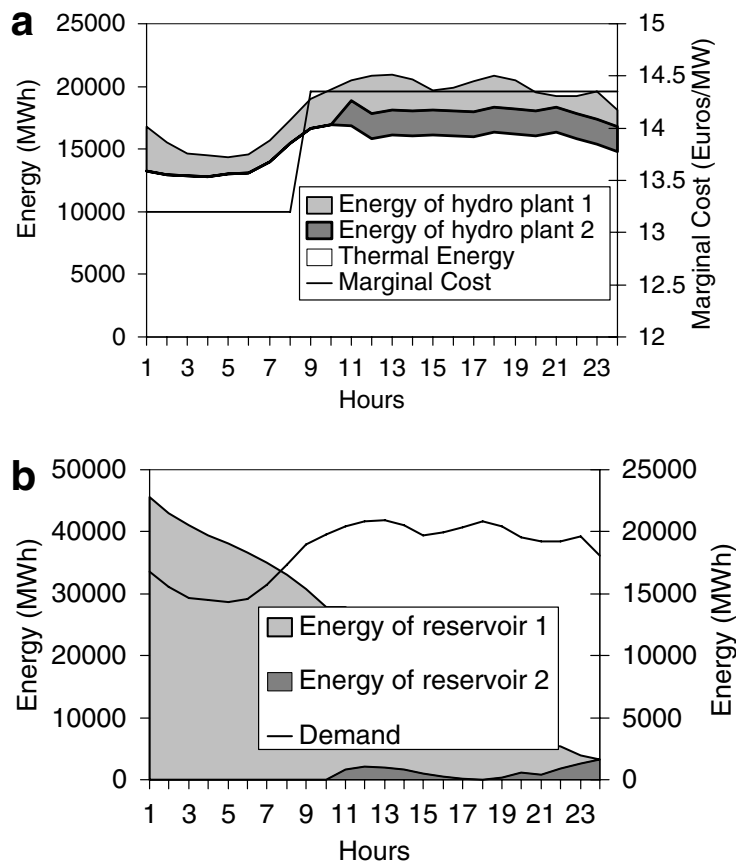

Fig. 12. Optimal thermal and hydraulic generation of a case with two cascaded reservoirs and all energy initially stored in the upstream reservoir.

cases. The results obtained are given in Table 4 . It can be observed that the GA leads to better feasible minima for both systems, being rather notable in the real case. Obviously, the IP algorithm reveals much better performance in CPU time. In the IP algorithm, the number of fitness evaluations means the number of total iterations since the problem is solved twice: first, adding the constraints (Eq. (13)) and later, considering the constraints (Eq. (14)).

Fig. 13 presents some differences between the solutions obtained from the GA and the IP algorithm for the thermal generators 4 and 5 in the small test system. Basically in both solutions the generators 1,2 and 3 are always on. The generators 4 and 5 are only on at minimum power during peak hours ( $7 \mathrm{am}$ to $11 \mathrm{am}$ and $7 \mathrm{am}$ to $12 \mathrm{pm}$, respectively) in the GA. However, in the solution obtained using
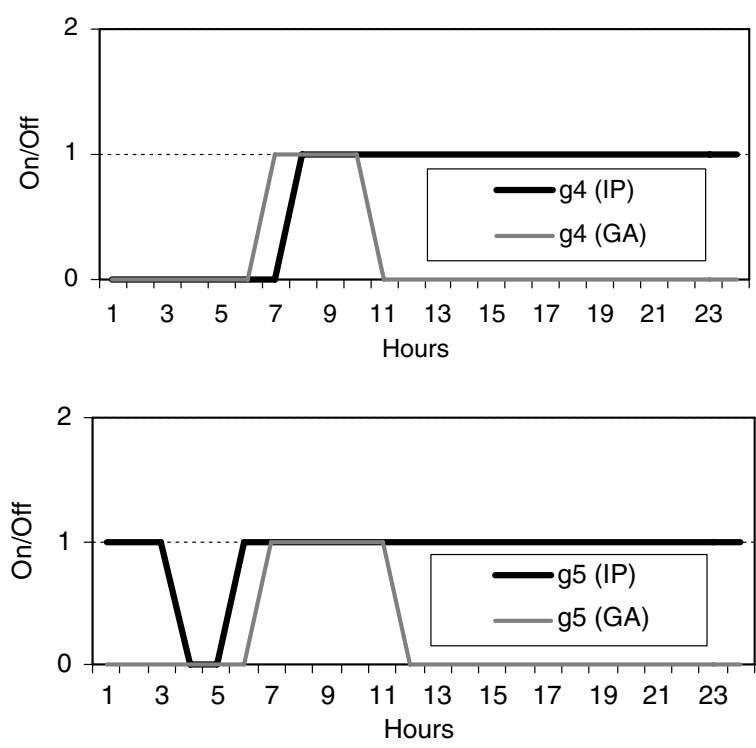

Fig. 13. Differences between the solutions obtained by the GA and the IP for the generators 4 and 5 .

an IP algorithm these generators are on at minimum power in most hours of the day (8am to 12am for the generator 4 and $1 \mathrm{am}$ to $3 \mathrm{am}$ and $6 \mathrm{am}$ to $12 \mathrm{am}$ for the generator 5). The produced energy for the generator 3 is adjusted to satisfy the demand depending on the state of the generators 4 and 5 as can be observed in Fig. 14.

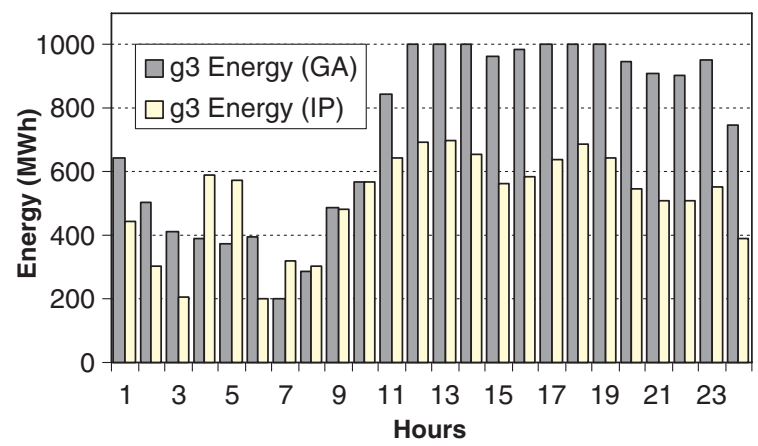

Fig. 14. Differences between the solutions obtained by the GA and the IP for the generator 3 .

Table 4

Comparison of GA and IP in terms of the objective function $C_{T}$, the number of fitness evaluations and the relative CPU time

\begin{tabular}{|c|c|c|c|c|c|c|}
\hline & \multicolumn{2}{|c|}{ Objective function (Euros) } & \multicolumn{2}{|c|}{ Number of fitness evaluations } & \multicolumn{2}{|c|}{ Relative CPU time } \\
\hline & GA & IP & GA & IP & GA & IP \\
\hline Test system & 528,832 & 594,407 & 100,000 & 37 & 737 & 1 \\
\hline Real system & $4,708,849$ & $4,881,891$ & 500,000 & 48 & 2800 & 1 \\
\hline
\end{tabular}


Table 5

Convergence of the IP algorithm

\begin{tabular}{|c|c|c|c|c|c|c|}
\hline \multirow[t]{2}{*}{ Iteration } & \multicolumn{3}{|c|}{ Constraints $0 \leqslant U_{i, t} \leqslant 1$} & \multicolumn{3}{|c|}{ Constraints $U_{i, t} \cdot\left(1-U_{i, t}\right)=0$} \\
\hline & $\mu$ & $\alpha$ & dugap & $\mu$ & $\alpha$ & dugap \\
\hline 1 & $1.000 \mathrm{E}+04$ & 0.35 & $9.982 \mathrm{E}+03$ & $1.000 \mathrm{E}+03$ & 0.23 & $9.974 \mathrm{E}+02$ \\
\hline 2 & $8.984 \mathrm{E}+03$ & 0.27 & $9.678 \mathrm{E}+03$ & $9.974 \mathrm{E}+01$ & 0.30 & $7.270 \mathrm{E}+02$ \\
\hline 3 & $8.711 \mathrm{E}+03$ & 0.39 & $9.278 \mathrm{E}+03$ & $7.270 \mathrm{E}+01$ & 0.64 & $3.103 \mathrm{E}+02$ \\
\hline 4 & $8.351 \mathrm{E}+03$ & 0.72 & $8.473 \mathrm{E}+03$ & $3.103 \mathrm{E}+01$ & 0.78 & $9.312 \mathrm{E}+01$ \\
\hline 5 & $7.625 \mathrm{E}+03$ & 0.99 & $7.603 \mathrm{E}+03$ & $9.312 \mathrm{E}+00$ & 0.99 & $1.027 \mathrm{E}+01$ \\
\hline 6 & $7.603 \mathrm{E}+02$ & 0.99 & $8.281 \mathrm{E}+02$ & $1.027 \mathrm{E}+00$ & 0.99 & $1.120 \mathrm{E}+00$ \\
\hline 7 & $8.281 \mathrm{E}+01$ & 0.99 & $9.030 \mathrm{E}+01$ & $1.120 \mathrm{E}-01$ & 0.99 & $1.221 \mathrm{E}-01$ \\
\hline 8 & $9.030 \mathrm{E}+00$ & 0.99 & $9.846 \mathrm{E}+00$ & $1.221 \mathrm{E}-02$ & 0.99 & $1.331 \mathrm{E}-02$ \\
\hline 9 & $9.846 \mathrm{E}-01$ & 0.99 & $1.074 \mathrm{E}+00$ & $1.331 \mathrm{E}-03$ & 0.99 & $1.452 \mathrm{E}-03$ \\
\hline 10 & $1.074 \mathrm{E}-01$ & 0.99 & $1.170 \mathrm{E}-01$ & $1.452 \mathrm{E}-04$ & 0.88 & $3.026 \mathrm{E}-04$ \\
\hline 11 & $1.171 \mathrm{E}-02$ & 0.99 & $1.275 \mathrm{E}-02$ & $3.026 \mathrm{E}-05$ & 0.75 & $9.822 \mathrm{E}-05$ \\
\hline 12 & $1.275 \mathrm{E}-03$ & 0.99 & $1.383 \mathrm{E}-03$ & $9.822 \mathrm{E}-06$ & 0.45 & $5.863 \mathrm{E}-05$ \\
\hline 13 & $1.380 \mathrm{E}-04$ & 0.77 & $4.244 \mathrm{E}-04$ & $5.863 \mathrm{E}-06$ & 0.34 & $4.066 \mathrm{E}-05$ \\
\hline 14 & $3.820 \mathrm{E}-04$ & 0.99 & $1.912 \mathrm{E}-04$ & $4.066 \mathrm{E}-06$ & 0.35 & $2.796 \mathrm{E}-05$ \\
\hline 15 & $1.900 \mathrm{E}-05$ & 0.88 & $3.225 \mathrm{E}-05$ & $2.796 \mathrm{E}-06$ & 0.20 & $2.283 \mathrm{E}-05$ \\
\hline 16 & $2.900 \mathrm{E}-05$ & 0.99 & $1.461 \mathrm{E}-05$ & $2.283 \mathrm{E}-06$ & 0.54 & $1.168 \mathrm{E}-05$ \\
\hline 17 & $1.000 \mathrm{E}-06$ & 0.56 & $6.810 \mathrm{E}-06$ & $1.168 \mathrm{E}-06$ & 0.48 & $6.676 \mathrm{E}-06$ \\
\hline 18 & & & & $6.676 \mathrm{E}-07$ & 0.10 & $6.049 \mathrm{E}-06$ \\
\hline 19 & & & & $6.049 \mathrm{E}-07$ & 0.24 & $4.735 E-06$ \\
\hline 20 & & & & $4.735 \mathrm{E}-07$ & 0.20 & $3.895 \mathrm{E}-06$ \\
\hline 21 & & & & $3.895 \mathrm{E}-07$ & 0.26 & $2.987 \mathrm{E}-06$ \\
\hline 22 & & & & $2.987 \mathrm{E}-07$ & 0.37 & $1.984 \mathrm{E}-06$ \\
\hline 23 & & & & $1.984 \mathrm{E}-07$ & 0.43 & $1.220 \mathrm{E}-06$ \\
\hline 24 & & & & $1.220 \mathrm{E}-07$ & 0.28 & $9.084 \mathrm{E}-07$ \\
\hline 25 & & & & $9.084 \mathrm{E}-08$ & 0.22 & $7.264 \mathrm{E}-07$ \\
\hline 26 & & & & $7.264 \mathrm{E}-08$ & 0.29 & $5.369 \mathrm{E}-07$ \\
\hline 27 & & & & $5.369 \mathrm{E}-08$ & 0.30 & $3.926 \mathrm{E}-07$ \\
\hline 28 & & & & $3.926 \mathrm{E}-08$ & 0.39 & $2.543 \mathrm{E}-07$ \\
\hline 29 & & & & $2.543 \mathrm{E}-08$ & 0.65 & $1.043 \mathrm{E}-07$ \\
\hline 30 & & & & $1.043 \mathrm{E}-08$ & 0.67 & $4.090 \mathrm{E}-08$ \\
\hline 31 & & & & $4.090 \mathrm{E}-09$ & 0.24 & $3.200 \mathrm{E}-08$ \\
\hline
\end{tabular}

Finally, Table 5 shows the convergence of the IP algorithm for the case based on the real system. First the problem is solved including the constraints (Eq. (13)) only to obtain an initialization point. Consequently, in this case the stopping criteria are not hard and 17 iterations are enough. Later, the same problem is solved with the constraints (Eq. (14)) using the former solution as initialization. In this case the stopping criteria must be more strict and 31 iterations have been need. Notice that the penalty factor and the average duality gap approach zero throughout the iterations.

\section{Conclusions}

In this paper, an evolutionary technique applied to the optimal short-term $(24 \mathrm{~h})$ electric energy production scheduling has been proposed. The equations defining the model of the problem have been presented leading to a non-linear mixed-inte- ger programming problem with a large number of continuous and integer variables. Some heuristics have been introduced to assure the feasibility of the solutions obtained by the GA, and key implementation issues have been discussed. Results from realistic cases based on the Spanish power system have been compared with that of a IP algorithm, revealing the good convergence characteristics and the remarkable performance of the proposed GA.

Further research will be addressed to improve the modelling of realistic cases to decrease the computational cost of the GA for real systems including hydroelectric power plants and to test other possible implementations of the selection, crossover and mutation operators maintaining the percentage of feasible individuals of the population. In the other hand, the GA and IP algorithms will be adapted to deal with multiple local minima in non-convex and non-linear optimization problems. 


\section{Acknowledgements}

The authors would like to acknowledge the financial support of the Spanish Ministry of Science and Technology, Project TIN2004-00159, and of the Junta de Andalucía, Projects ACPAI-2003/032 and P05-TIC-00531.

\section{References}

[1] A.J. Wood, B.F. Wollenberg, Power Generation, Operation and Control, John Wiley and Sons Inc., 1996.

[2] N. Prasad Padhy, Unit commitment - a bibliographical survey, IEEE Transactions on Power Systems 19 (2) (2004) 1196-1205.

[3] J.M. Arroyo, A.J. Conejo, A parallel repair genetic algorithm to solve the unit commitment problem, IEEE Transactions on Power Systems 17 (4) (2002) 1216-1224.

[4] I.G. Damousis, A.G. Bakirtzis, P.S. Dokopoulos, A solution to the unit-commitment problem using integer-coded genetic algorithm, IEEE Transactions on Power Systems 19 (2) (2004) 1165-1172.

[5] A. Forsgren, P.E. Gill, M.H. Wright, Interior point methods for nonlinear optimization, SIAM Review 44 (4) (2002) 525597.

[6] M. Argáez, R.A. Tapia, On the global convergence of a modified augmented Lagrangian linesearch Interior-Point Newton method for nonlinear programming, Journal of Optimization Theory and Applications 114 (1) (2001) 1-25.

[7] H.Y. Benson, R.J. Vanderbei, D. Shannno, Interior-point methods for nonconvex nonlinear programming: Filter methods and merit functions, Computational Optimization and Applications 23 (2) (2002) 257-272.

[8] R.H. Byrd, J. Nocedal, R.A. Waltz, Feasible interior methods using slacks for nonlinear optimization, Computational Optimization and Applications 26 (1) (2003) 35-61.

[9] H. Yamashita, H. Yabe, Quadratic convegence of a primaldual interior point method for degenerate nonlinear optimization problems, Computational Optimization and Applications 31 (2) (2005) 123-143.

[10] P. Tseng, Convergent infeasible Interior-point trust-region methods for constrained minimization, SIAM Journal on Optimization 13 (2) (2002) 432-469.

[11] M. Liu, S.K. Tso, Y. Cheng, An extended nonlinear primaldual Interior-point algorithm for reactive-power optimization of large-scale power systems with discrete control variables, IEEE Transactions on Power Systems 17 (4) (2002) 982-991.

[12] Y. Li, X. Zon, L. Kang, Z. Michalewicz, A new dynamical evolutionary algorithm based on statistical mechanics, Journal of Computer Science and Technology 18 (3) (2003) 361-368.

[13] C.C.A. Rajan, M.R. Mohan, An evolutionary programming-based Tabu search method for solving the unit commitment problem, IEEE Transactions on Power Systems 19 (1) (2004) 577-585.

[14] S. Janson, M. Middendorf, A hierarchical particle swarm optimizer and its adaptive variant, IEEE Transactions on Systems, Man, and Cybernetics - Part B: Cybernetics 35 (6) (2005) 1272-1282.

[15] W. Ben-Ameur, Computing the initial temperatura of simulated annealing, Computational Optimization and Applications 29 (3) (2005) 369-385.

[16] J. Dréo, P. Siarry, A new ant colony algorithm using the heterarchical concept aimed at optimization of multiminima continuous functions, Lecture Notes in Computer Science 2463 (2002) 216-221.

[17] R. Chelouah, P. Siarry, A hybrid method combining continuous Tabu search and Nelder-Mead simplex algorithms for the global optimization of multiminima functions, European Journal of Operational Research 161 (3) (2005) 636-654.

[18] S. Kannan, S.M.R. Slochanal, N. Prasad Padhy, Application and comparison of metaheuristic techniques to generation expansion planning problem, IEEE Transactions on Power Systems 20 (1) (2005) 466-475.

[19] W.F. Tinney, V. Brandwajn, Chan, Sparse vector methods, IEEE Transactions on Power Systems 104 (1985) 295-301.

[20] D.E. Goldberg, Genetic Algorithms in Search, Optimization and Machine Learning, Addison-Wesley, Ready, Massachusetts, USA, 1989.

[21] Z. Michalewicz, Genetic Algorithms + Data Structures $=$ Evolution Programs, third ed., Springer-Verlag, Berlin, Germany, 1996.

[22] Victor H. Quintana, Geraldo L. Torres, Jose MedinaPalomo, Interior-point methods and their applications to power systems: A classification of publications and software codes, IEEE Transactions on Power Systems 15 (1) (2000) $170-176$

[23] H. Yamin, S. Al-Agtash, M. Shahidehpour, Security-constrained optimal generation scheduling for GENCOs, IEEE Transactions on Power Systems 19 (3) (2004) 13651372. 